\title{
Platelet Microparticles: Making Blood a Bad Humor
}

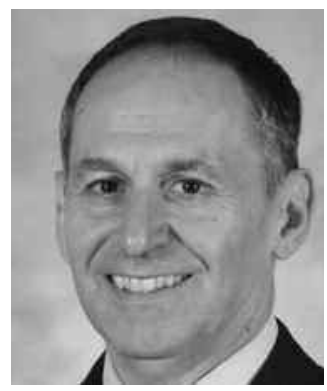

Ancient physicians based their practice of medicine on the humoral theory of disease. This theory posited that substances called humors make up the body and determine health and disease. When in balance, these humors - blood, yellow bile, black bile, and phlegm - caused health; out of balance, they caused disease. This theory also related humors to the 4 elements: earth, fire, water, and air. Of the humors, blood was unique in its content of all 4 elements.

Now relegated to the realm of quaint and discredited ideas of the past, the humoral theory of disease nevertheless has elements of truth. Importantly, this theory recognized the importance of blood in disease pathogenesis and the diversity of bodily constituents that can influence disease. With scientific advance, earth, fire, water, and air have been replaced by cells, cytokines, chemokines, and prostanoids, among many others. The list of these substances is ever increasing, with disciplines of genomics, proteomics, metabolomics, and nucleomics filling the blood to the brim with noxious mediators. Reading today's literature, we can be wistful about a time when physicians could understand disease with only 4 elements, in contrast to today's 4 thousand or even 4 million.

As an article in this issue of The Journal demonstrates, the blood of patients with connective tissue disease (CTD) has novel elements that can drive disease and serve as biomarkers to elucidate pathogenesis and assess disease activity ${ }^{1}$. Thus, using an enzyme-lined immunosorbent assay (ELISA), Oyabu, et al provide evidence that the blood of patients with CTD has increased levels of platelet-derived microparticles (PDMP) and that elevations of PDMP are related to Raynaud's phenomenon (RP). This article is important in substantiating the value of solid-phase methods to detect particles and in delineating clinical associations.

As shown in in vivo and in vitro studies, microparticles are potentially important actors in disease pathogenesis because of their proinflammatory and prothrombotic activities. These structures are small, membrane-bound vesicles that are released from activated or dying cells, incorporating nuclear and cytoplasmic constituents as they form by a blebbing process. In blebbing, the cell membrane forms bubble-like structures especially during apoptosis as the cell shrinks. As blebs form at the cell surface, some detach to enter the blood. The process is important since particles have potent signaling activity in intercellular communication and may even transfer bioactive molecules including genetic information ${ }^{2}$.

Of particles in the blood, those derived from platelets are usually the most abundant. Originally called platelet dust, PDMP are released during platelet activation and can promote thrombosis because of their display of phosphatidylserine (PS). PS is ordinarily on the inner leaflet of the cell membrane, although it can translocate to the outside when platelets undergo activation; PS translocation also occurs during apoptosis because lipid asymmetry breaks down. Once on a particle or cell surface, PS can promote thrombosis via the assembly of coagulation complexes ${ }^{3,4}$. In addition, microparticles have immune activity and can interact with immune cells to promote immune responses ${ }^{5,6}$.

Not surprisingly, increased numbers of microparticles occur in diseases characterized by vasculopathy, including atherosclerosis, diabetes, and stroke, among many others. While platelets (and megakaryocytes) are a major source of particles in these conditions, microparticles can originate from many cell types, including immune cells and endothelium. Indeed, depending on the clinical condition, the population of blood particles can be highly diverse, and it is likely that all particle types can promote immune activation and thrombosis. Given the wide expression of microparticles in disease, these structures have attracted great interest as novel elements in pathogenesis?

While the biology of microparticles is increasingly well defined, their assay remains problematic. Flow cytometry (FACS) is the most commonly used method for particle detection, measuring particles by light scatter or staining of particles for cell-surface markers. These approaches, while

See Plasma platelet-derived microparticles in patients with CTD, page 680 
useful, are nevertheless limited. Microparticles are much smaller than cells and usually range in size from 0.1 to 1.0 $\mu \mathrm{m}$. As such, data acquisition is tricky and requires the use of logarithmic gain for detection. Since particles overlap "noise" that is electronic in origin, using machine settings to reduce noise limits detection, especially involving particles at the low end of the size range. The number of particles not counted is not known ${ }^{8}$.

To bypass the problems of light scattering, many studies detect particles by FACS using staining with cell-surface markers since, as particles detach from cells, they bear these markers. In addition, because particle membranes express PS, particles can bind annexin V to allow detection. These immunochemical approaches, while allowing more unambiguous particle detection, also have limitations. The surface area of particles is much smaller than that of a cell; staining by antibodies or annexin V is thus low. Further, membrane markers may not translocate to particles in adequate numbers for detection and many particles are in fact annexin $\mathrm{V}$-negative. Whether the failure to bind annexin $\mathrm{V}$ reflects a distinct mode of particle release is unknown.

Rather than using FACS, Oyabu, et al used a sandwichtype ELISA based on the interaction of platelet-free plasma to plates coated with an anti-GPIX antibody to bind PDMP using a peroxidase-conjugated anti-GPIb antibody for detection. Prior studies have shown that ELISA assays correlate well with FACS determinations, although they require particles that simultaneously express 2 platelet markers ${ }^{9,10}$. Since PDMP display heterogeneity in terms of size and marker expression, an ELISA, like any other detection method, may miss particle subpopulations. The advantages of an ELISA are nevertheless significant, although issues of sample preparation (e.g., collection buffer, centrifugation speed, freeze-thawing, sample storage, etc.) remain ${ }^{11}$.

The results of the current study are consistent with others in demonstrating the presence of increased numbers of PDMP with CTD ${ }^{12,13,14}$. Thus, PDMP levels were elevated in blood from patients with mixed CTD, polymyositis/dermatomyositis (PM/DM), systemic sclerosis, and systemic lupus erythematosus (SLE). Despite trends with the samples from SLE and PM/DM patients, the values did not differ significantly from controls. The findings with SLE are surprising in view of prior studies, although these differences could relate to the assays used, patient population, and disease activity.

In a further analysis of data, PDMP elevations were associated with Raynaud's phenomenon (RP). This finding is interesting in view of the postulated role of thrombosis in the vasculopathy of RP, although the interpretation of the findings bears discussion. RP is highly heterogeneous, ranging from annoying but transient episodes of vasospasm in the cold to devastating ischemic events with infarction. In general, problems with RP tend to be much more severe in systemic sclerosis than in SLE or rheumatoid arthritis ${ }^{15,16}$.
Since some patients may consider tingling or pain in the cold as RP, relying on patient self-reports for ascertainment, as was done in this study, can be problematic.

In addition, the time element is important in evaluating the mechanistic and biomarker significance of PDMP elevations. In the current study by Oyabu, et al, patients were included in the study if RP was present in the month prior to blood drawing, although, as shown for selected patients, PDMP levels appeared to be chronically elevated. Many of these patients were treated with agents that could potentially influence vascular events, although the clinical response is not known. Together, these findings could suggest that, in patients with RP, platelet activation is ongoing and may not be directly related to the ischemic events themselves nor be ameliorated by treatment. A study during a clinically apparent episode would have been informative to clarify the role of particles in the vasculopathy.

PDMP are but one element in the blood but they are heterogeneous, and their properties may be mutable as proteins cover their surface $17,18,19,20$. Since these proteins, especially autoantibodies, can vary in the blood of patients with CTD, the functional consequences of elevated PDMP numbers may differ depending on the platelet covering and the total structural ensemble. It is therefore premature to conclude that the effects of PDMP extend across the disease spectrum, especially if both the intrinsic and extrinsic protein components of the particles are different.

The study of Oyabu, et al provides new insights into the role of microparticles in the pathogenesis of CTD, with elevations of PDMP levels in patients with RP evidence of ongoing vasculopathy. Further, these findings suggest the utility of PDMP determinations in subsetting patients on the basis of clinical features as well as diagnosis. As the Greek and Roman physicians postulated, blood is unique because of its content of the elements. Microparticles were not part of their lexicon. Had they been, the ancient physicians would have no doubt identified these structures as a key component in the body and, indeed, an element that can turn blood from a vital fluid into a truly bad humor.

\author{
DAVID S. PISETSKY, MD, PhD, \\ Medical Research Service, \\ Durham Veterans Administration Medical Center, \\ Duke University Medical Center, \\ Durham, North Carolina, USA
}

Address correspondence to Dr. D.S. Pisetsky, $151 G$ Durham VA Medical Center, 508 Fulton Street, Durham, NC 27705, USA. E-mail address:dpiset@acpub.duke.edu

\section{REFERENCES}

1. Oyabu C, Morinobu A, Sugiyama D, Saegusa J, Tanaka S, Morinobu S, et al. Plasma platelet-derived microparticles in patients with connective tissue diseases. J Rheumatol 2011;38:680-4.

2. Beyer C, Pisetsky DS. The role of microparticles in the 
pathogenesis of rheumatic diseases. Nat Rev Rheumatol 2010;6:21-9.

3. Morel O, Morel N, Freyssinet JM, Toti F. Platelet microparticles and vascular cells interactions: a checkpoint between the haemostatic and thrombotic responses. Platelets 2008;19:9-23.

4. Italiano JE Jr, Mairuhu AT, Flaumenhaft R. Clinical relevance of microparticles from platelets and megakaryocytes. Curr Opin Hematol 2010;17:578-84.

5. Boilard E, Nigrovic PA, Larabee K, Watts GFM, Coblyn JS, Weinblatt ME, et al. Platelets amplify inflammation in arthritis via collagen-dependent microparticle production. Science 2010;327:580-3.

6. Pisetsky DS, Lipsky PE. Microparticles as autoadjuvants in the pathogenesis of SLE. Nat Rev Rheumatol 2010;6:368-72.

7. VanWijk MJ, VanBavel E, Sturk A, Nieuwland R. Microparticles in cardiovascular diseases. Cardiovasc Res 2003;59:277-87.

8. Orozco AF, Lewis DE. Flow cytometric analysis of circulating microparticles in plasma. Cytometry A 2010;77A:502-14.

9. Osumi K, Ozeki Y, Saito S, Nagamura Y, Ito H, Kimura Y, et al. Development and assessment of enzyme immunoassay for platelet-derived microparticles. Thromb Haemost 2001;85:326-30.

10. Nomura S, Shouzu A, Taomoto K, Togane Y, Goto S, Ozaki Y, et al. Assessment of an ELISA kit for platelet-derived microparticles by joint research at many institutes in Japan. J Atheroscler Thromb 2009;16:878-87.

11. Shah MD, Bergeron AL, Dong J-F, Lopez JA. Flow cytometric measurement of microparticles: pitfalls and protocol modifications. Platelets 2008;19:365-72.

12. Pereira J, Alfaro G, Goycoolea M, Quiroga T, Ocqueteau M, Massardo L, et al. Circulating platelet-derived microparticles in systemic lupus erythematosus. Association with increased thrombin generation and procoagulant state. Thromb Haemost 2006;95:94-9.
13. Guiducci S, Distler JH, Jüngel A, Huscher D, Huber LC, Michel $\mathrm{BA}$, et al. The relationship between plasma microparticles and disease manifestations in patients with systemic sclerosis. Arthritis Rheum 2008;58:2845-53.

14. Sellam J, Proulle V, Jüngel A, Ittah M, Richard CM, Gottenberg $\mathrm{J}-\mathrm{E}$, et al. Increased levels of circulating microparticles in primary Sjögren's syndrome, systemic lupus erythematosus and rheumatoid arthritis and relation with disease activity. Arthritis Res Ther 2009;11:1-11.

15. Herrick AL. Pathogenesis of Raynaud's phenomenon. Rheumatology 2005;44:587-96.

16. Block JA, Sequeira W. Raynaud's phenomenon. Lancet 2001;357:2042-8.

17. Perez-Pujol S, Marker PH, Key NS. Platelet microparticles are heterogeneous and highly dependent on the activation mechanism: studies using a new digital flow cytometer. Cytometry A 2007;71A:38-45.

18. Dean WL, Lee MJ, Cummins TD, Schultz DJ, Powell DW. Proteomic and functional characterization of platelet microparticle size classes. Thromb Haemost 2009;102:711-8.

19. Biró E, Nieuwland R, Tak PP, Pronk LM, Schaap MC, Sturk A, et al. Activated complement components and complement activator molecules on the surface of cell-derived microparticles in patients with rheumatoid arthritis and healthy individuals. Ann Rheum Dis 2007;66:1085-92.

20. Dasgupta SK, Abdel-Monem H, Niravath P, Le A, Bellera R, Langlois K, et al. Lactadherin and clearance of platelet-derived miscrovesicles. Platelets Thromb 2009;113:1332-9.

J Rheumatol 2011;38:590-2; doi:10.3899/jrheum.101359 